PROCEEDINGS OF THE

AMERICAN MATHEMATICAL SOCIETY

Volume 134, Number 1, Pages 139-148

S 0002-9939(05)07780-4

Article electronically published on August 15, 2005

\title{
ON THE BERGMAN METRIC OF PSEUDOCONVEX DOMAINS IN A COMPLEX PROJECTIVE SPACE
}

\author{
BO-YONG CHEN
}

(Communicated by Mei-Chi Shaw)

\begin{abstract}
We prove a localization principle of the Bergman kernel form and metric for $C^{2}$ pseudoconvex domains in the complex projective space. An estimate of the Bergman distance is also given.
\end{abstract}

\section{INTRODUCTION}

Let $M$ be a complex $n$-dimensional manifold. Let $\mathcal{H}$ be the Hilbert space of holomorphic $n$-forms on $M$ such that $\left|\int_{M} f \wedge \bar{f}\right|<\infty$. Let $h_{0}, h_{1}, \cdots$ be a complete orthonormal basis for $\mathcal{H}$. Then the $2 n$-form defined on $M \times M$ given by $K_{M}=\sum_{j=0}^{\infty} h_{j} \wedge \bar{h}_{j}$ is called the Bergman kernel of $M$. Let $\left(z_{1}, \cdots, z_{n}\right)$ be a local coordinate system in $M$ and let $K_{M}(z)=K_{M}^{*}(z) d z_{1} \wedge \cdots \wedge d z_{n} \wedge d \bar{z}_{1} \wedge \cdots \wedge d \bar{z}_{n}$ where $K_{M}^{*}$ is a locally defined function. Thus $\beta_{M}:=\partial \bar{\partial} \log K_{M}^{*}$ is a well-defined Hermitian form of bidegree $(1,1)$, whenever $K_{M}^{*}$ is nonzero. We say that $M$ possesses a Bergman metric iff $\beta_{M}$ is everywhere positive definite. The following localization principle for the Bergman kernel (metric) is well known.

Theorem (cf. [17, 9]). Let $\Omega \subset \subset \mathbf{C}^{n}$ be pseudoconvex. Then for any two neighborhoods $V \subset \subset U$ of a boundary point $p$ there is a constant $C>0$ such that

$$
\begin{aligned}
K_{\Omega}(x) & \geq C \cdot K_{\Omega \cap U}(x), \\
\beta_{\Omega}(x ; X) & \geq C \cdot \beta_{\Omega \cap U}(x ; X)
\end{aligned}
$$

for all $x \in \Omega \cap V$ and $X \in T_{x}^{1,0}(\Omega)$.

It is natural to ask on which pseudoconvex domains in $\mathbf{P}^{n}$ the localization principle holds. (Notice that the complement of a hypersurface in $\mathbf{P}^{n}$ is pseudoconvex; however, the Bergman kernel form vanishes.) In this direction, Diederich and Ohsawa 11] proved the localization principle for another Bergman kernel (metric), which is induced by square-integrable holomorphic functions with respect to the Fubini-Study metric on any pseudoconvex domain $\Omega$ in $\mathbf{P}^{n}$ such that the interior of its complement is not empty. Such a Bergman metric is not invariant under biholomorphic mappings. In this note, we will show

Received by the editors January 23, 2004.

2000 Mathematics Subject Classification. Primary 32A25.

Key words and phrases. Bergman kernel form, Bergman metric, complex projective space.

This work was supported by JSPS. 
Theorem 1. Let $\Omega$ be a pseudoconvex domain with $C^{2}$ boundary in $\mathbf{P}^{n}$. Then there is a constant $\gamma>0$ such that for any two neighborhoods $V \subset \subset U$ of a boundary point $p$, one has

$$
\begin{aligned}
\left|K_{\Omega}(x)\right|_{F S} & \geq C \cdot \frac{\left|K_{\Omega \cap U}(x)\right|_{F S}}{\left|\log \delta_{\Omega}(x)\right|^{\gamma}}, \\
\beta_{\Omega}(x ; X) & \geq C \cdot \frac{\beta_{\Omega \cap U}(x ; X)}{\left|\log \delta_{\Omega}(x)\right|^{\gamma}}
\end{aligned}
$$

for all $x \in \Omega \cap V$ and $X \in T_{x}^{1,0}(\Omega)$. Here $\delta_{\Omega}$ (resp. $|\cdot|_{F S}$ ) denotes the boundary distance (resp. length) w.r.t. the Fubini-Study metric $d s_{F S}^{2}$.

As an immediate consequence of Theorem 1 and the Ohsawa-Takegoshi extension theorem [19], we obtain

Corollary. Let $\Omega$ be as above. Then

$$
\left|K_{\Omega}(x)\right|_{F S} \geq C \cdot \delta_{\Omega}(x)^{-2}\left|\log \delta_{\Omega}\right|^{-\gamma} .
$$

We mention that Theorem 1 cannot be generalized to an arbitrary fat pseudoconvex domain in $\mathbf{P}^{n}$ as the following example shows.

Example. We consider the following domain:

$$
\Omega=\left\{\left[z_{0}, z_{1}, z_{2}\right] \in \mathbf{P}^{2}:\left|z_{1}\right|<\left|z_{0}\right|\right\}
$$

where $\left(z_{0}, z_{1}, z_{2}\right)$ denote homogeneous coordinates in $\mathbf{P}^{2}$. Note that $\Omega$ is biholomorphic to the product of the unit disc and the complex plane via

$$
\zeta_{1}=z_{1} / z_{0}, \zeta_{2}=z_{2} / z_{0} .
$$

Hence $\Omega$ is a fat pseudoconvex domain in $\mathbf{P}^{n}$, but the Bergman metric is degenerate.

However, the estimate of the Bergman metric in Theorem 1 does not imply the completeness. It was shown in 5 that every hyperconvex manifold is Bergman complete, while every $C^{2}$ pseudoconvex domain in $\mathbf{P}^{n}$ is hyperconvex [18]; hence it is Bergman complete. We mention that there are a lot of works concerning the completeness of $\beta_{M}$ (cf. [4], [5], 6], 12, 13, [14, 15], 17, 20]). On the other hand, Diederich and Ohsawa [10] proved that the Bergman distance for a bounded $C^{2}$ pseudoconvex domain in $\mathbf{C}^{n}$ has a lower bound of a constant multiple $\log \left|\log \delta_{\Omega}\right|$. This lower bound was improved by Blocki 3] to $\left|\log \delta_{\Omega}\right| / \log \left|\log \delta_{\Omega}\right|$. We extend his result to the complex projective space.

Theorem 2. Let $\Omega \subset \mathbf{P}^{n}$ be a pseudoconvex domain with $C^{2}$ boundary. Then

$$
\operatorname{dist}_{\Omega}\left(x_{0}, x\right) \geq \frac{C\left|\log \delta_{\Omega}(x)\right|}{\log \left|\log \delta_{\Omega}(x)\right|},
$$

where $\operatorname{dist}_{\Omega}\left(x_{0}, x\right)$ denotes the Bergman distance between $x_{0}$ and $x$.

\section{Proof of Theorem 2}

Let $y \in \Omega$ be arbitrary fixed. Take a smooth function $\kappa: \mathbf{R} \rightarrow[0,1]$ such that $\left.\kappa\right|_{(-\infty, 1 / 2]}=1$ and $\left.\kappa\right|_{[1, \infty)}=0$. Let $d_{F S}(x, y)$ denote the Fubini-Study distance between two points $x, y$ in $\mathbf{P}^{n}$. Let $r$ be the injectivity radius of $\mathbf{P}^{n}$ and $c$ be the upper bound of the sectional curvature. Set

$$
\phi_{y}(x)=\kappa\left(d_{F S}(x, y) / r_{0}\right) \log \left(d_{F S}(x, y) / r_{0}\right)-1
$$


where $r_{0}=\min \{r, \pi / 2 c\}$. By the Hessian comparison theorem (cf. [12]), there is a positive constant $C_{1}$ independent of $y$ such that

$$
\partial \bar{\partial} \phi_{y}(x) \geq-C_{1} d s_{F S}^{2} .
$$

From now on we assume $r_{0}=1$ for the sake of simplicity. By [18, there exists a smooth and strictly psh function $\rho: \Omega \rightarrow(-1,0)$ such that $|\rho| \approx \delta_{\Omega}^{\tau}$ for some $0<\tau<1$ and

$$
\partial \bar{\partial} \rho \geq C_{2}|\rho| d s_{F S}^{2}
$$

where $C_{2}$ is a positive constant. Choose a cut-off function $\chi: \mathbf{R} \rightarrow[0,1]$ such that $\chi \equiv 1$ on $[-1 / 2,1 / 2]$ and $\chi \equiv 0$ on $[1,+\infty) \cup(-\infty,-1]$. For any $y \in M$, we set

$$
\varphi_{y}=\chi(\log (-\log (-\rho))-\log (-\log (-\rho(y)))) \phi_{y} .
$$

Following an idea of [10, we will show

Lemma 3. There exists a sufficiently large constant $b$ such that for any $y \in \Omega$ satisfying $|\rho(y)|<2^{-e}$, we have

(i) $\varphi_{y}-\log \left(-\phi_{y}\right)-b \log (-\rho)$ is $C^{2}$ strictly psh on $\Omega \backslash\{y\}$;

(ii) $\varphi_{y}$ has a logarithmic pole at $y$;

(iii) $\operatorname{supp} \varphi_{y} \subset\left\{x \in \Omega:|\rho(y)|^{e} \leq|\rho(x)| \leq|\rho(y)|^{1 / e}\right\}$.

Proof. (ii), (iii) are trivial. We only need to show (i). By a straightforward computation, we obtain on $\Omega \backslash\{y\}$,

$$
\begin{aligned}
\partial \bar{\partial} \varphi_{y}= & \frac{\phi_{y}}{\log (-\rho)}\left(\chi^{\prime \prime}(\cdot) \frac{\partial \log (-\rho) \bar{\partial} \log (-\rho)}{\log (-\rho)}\right. \\
& \left.-\chi^{\prime}(\cdot) \frac{\partial \log (-\rho) \bar{\partial} \log (-\rho)}{\log (-\rho)}+\chi^{\prime}(\cdot) \partial \bar{\partial} \log (-\rho)\right) \\
& +\frac{\chi^{\prime}(\cdot) \phi_{y}}{\log (-\rho)}\left(\partial \log (-\rho) \frac{\bar{\partial} \phi_{y}}{\phi_{y}}+\frac{\partial \phi_{y}}{\phi_{y}} \bar{\partial} \log (-\rho)\right) \\
& +\chi(\cdot) \partial \bar{\partial} \phi_{y} .
\end{aligned}
$$

By the Cauchy-Schwarz inequality, for any constant $\theta>0$ one has

$$
\begin{aligned}
& \pm 2 \operatorname{Re}\left\{\partial \log (-\rho) \frac{\bar{\partial} \phi_{y}}{\phi_{y}}\right\} \\
\leq & \theta \partial \log (-\rho) \bar{\partial} \log (-\rho)+\theta^{-1} \partial \log \left(-\phi_{y}\right) \bar{\partial} \log \left(-\phi_{y}\right) .
\end{aligned}
$$

Since $-\rho(y)<2^{-e}$, it follows from $(i i i)$ that $-\rho(x)<1 / 2$ on $\operatorname{supp} \varphi_{y}$. Hence there is a positive constant $C_{3}$ depending only on the choice of $\chi$ such that

$$
\begin{aligned}
\partial \bar{\partial} \varphi_{y} \geq & -\frac{C_{3}\left|\phi_{y}\right|}{|\log (-\rho)|}\{\partial \bar{\partial}(-\log (-\rho))+\theta \partial \log (-\rho) \bar{\partial} \log (-\rho) \\
& \left.+\theta^{-1} \partial \log \left(-\phi_{y}\right) \bar{\partial} \log \left(-\phi_{y}\right)\right\}-C_{1} d s_{F S}^{2} .
\end{aligned}
$$

Note that

$$
\begin{aligned}
\operatorname{supp} \chi^{\prime}(\cdot) \subset \quad & \left\{x \in \Omega:|\rho(y)|^{e} \leq|\rho(x)| \leq|\rho(y)|^{\sqrt{e}}\right. \\
& \text { or } \left.|\rho(y)|^{1 / \sqrt{e}} \leq|\rho(x)| \leq|\rho(y)|^{1 / e}\right\} .
\end{aligned}
$$

This implies

$$
|\rho(x)-\rho(y)| \geq \frac{|\rho(x)|}{2}
$$


on $\operatorname{supp} \chi^{\prime}(\cdot)$. Since $|\rho| \approx \delta_{\Omega}^{\tau},|\rho(x)-\rho(y)|=O\left(d_{F S}(x, y)^{\tau}\right)$. By the above inequality, there is a constant $C_{4}>0$ independent of $y$ so that

$$
\left|\phi_{y}\right| \leq C_{4}|\log (-\rho)|
$$

holds on $\operatorname{supp} \chi^{\prime}(\cdot)$. On the other hand, one has

$$
\begin{aligned}
\partial \bar{\partial}(-\log (-\rho)) & =\frac{\partial \bar{\partial} \rho}{|\rho|}+\partial \log (-\rho) \bar{\partial} \log (-\rho) \\
& \geq C_{2} d s_{F S}^{2}+\partial \log (-\rho) \bar{\partial} \log (-\rho), \\
\partial \bar{\partial}\left(-\log \left(-\phi_{y}\right)\right) & =\frac{\partial \bar{\partial} \phi_{y}}{\left|\phi_{y}\right|}+\partial \log \left(-\phi_{y}\right) \bar{\partial} \log \left(-\phi_{y}\right) \\
& \geq-C_{1} d s_{F S}^{2}+\partial \log \left(-\phi_{y}\right) \bar{\partial} \log \left(-\phi_{y}\right)
\end{aligned}
$$

since $\phi_{y} \leq-1$. Hence after fixing sufficiently large $\theta$, we can choose $b>0$ such that $(i)$ holds. The proof is complete.

Let $g_{\Omega}$ be the pluricomplex Green function on $\Omega$, i.e., $g_{\Omega}(x, y)=\sup \{u(x)\}$ where the supremum is taken over all negative functions $u \in P S H(\Omega)$ satisfying the property that the function $u-\log |z|$ is bounded from above in a deleted neighborhood of $y$ for some holomorphic local coordinates $z$ centered at $y$, that is, $z(y)=0$.

Lemma 4. There is a constant $c>0$ such that

$$
g_{\Omega}(x, y)>-\frac{c \rho(x)}{\rho(y)} \cdot|\log (-\rho(y))|
$$

for any $x, y \in \Omega$ with $|\rho(x)| \leq|\rho(y)| / 2$.

Proof. Let $\epsilon=|\rho(y)|^{e}$ and set

$$
\lambda_{y}=\varphi_{y}-\log \left(-\phi_{y}\right)-b \log (-\rho)+2 b \log \epsilon .
$$

By Lemma $3, \lambda_{y}$ is a negative psh function on $\Omega_{\epsilon^{2}}=\left\{x \in \Omega:|\rho(x)|>\epsilon^{2}\right\}$. Set

$$
\eta_{y}= \begin{cases}\max \left\{\lambda_{y}, c_{y}\left(\rho+\epsilon^{2}\right)\right\}, & |\rho| \leq \epsilon, \\ \lambda_{y}, & |\rho|>\epsilon,\end{cases}
$$

where

$$
c_{y}=-\frac{1}{\epsilon-\epsilon^{2}} \cdot \inf _{|\rho(x)|=\epsilon} \lambda_{y}(x) .
$$

Then $\eta_{y}$ is a well-defined negative psh function on $\Omega_{\epsilon^{2}}$ such that

$$
\eta_{y}(x) \geq c_{y} \rho(x) \geq C_{5} \rho(x)|\log \epsilon| /\left(\epsilon-\epsilon^{2}\right) \geq-C_{6}
$$

for all $|\rho(x)| \leq \epsilon^{3 / 2}$. Here $C_{5}, C_{6}$ are positive constants independent of $y$. Set

$$
\tilde{\rho}=-C_{6} \frac{\log \left(-\rho+\epsilon^{2}\right)-\log \left(2 \epsilon^{2}\right)}{\log \left(\epsilon^{3 / 2}+\epsilon^{2}\right)-\log \left(2 \epsilon^{2}\right)} .
$$

Clearly, $\tilde{\rho}$ is a psh function on $\Omega$ such that $\tilde{\rho} \leq C_{7}$ where $C_{7}$ is independent of $\epsilon$. Note that

$$
\begin{aligned}
& \tilde{\rho}(x)=-C_{6} \leq \eta_{y}(x), \quad \text { if } \quad|\rho(x)|=\epsilon^{3 / 2}, \\
& \tilde{\rho}(x)=0=\eta_{y}(x), \quad \text { if } \quad|\rho(x)|=\epsilon^{2} .
\end{aligned}
$$


Hence the function defined by

$$
\mu_{y}= \begin{cases}\eta_{y}, & \text { on }\left\{|\rho|>\epsilon^{3 / 2}\right\}, \\ \max \left\{\eta_{y}, \tilde{\rho}\right\}, & \text { on }\left\{\epsilon^{2} \leq|\rho| \leq \epsilon^{3 / 2}\right\}, \\ \tilde{\rho}, & \text { on }\left\{|\rho|<\epsilon^{2}\right\}\end{cases}
$$

is well-defined, psh on $\Omega$ and has a logarithmic pole at $y$. Set

$$
\nu_{y}= \begin{cases}\max \left\{\mu_{y}-C_{7}, \tilde{c}_{y} \rho\right\}, & \rho(x) \geq \frac{1}{2} \rho(y), \\ \mu_{y}-C_{7}, & \rho(x)<\frac{1}{2} \rho(y),\end{cases}
$$

where

$$
\tilde{c}_{y}=\frac{2}{\rho(y)} \inf _{\rho(x)=\frac{1}{2} \rho(y)}\left(\mu_{y}(x)-C_{7}\right) .
$$

If $\rho(x) \geq \frac{1}{2} \rho(y)$, then we have

$$
\frac{|\rho(y)|}{2} \leq|\rho(x)-\rho(y)| \leq c^{\prime} \cdot d_{F S}(x, y)^{\tau}
$$

for a suitable constant $c^{\prime}>0$, which implies

$$
\tilde{c}_{y} \leq \frac{C_{8}|\log (-\rho(y))|}{|\rho(y)|} .
$$

Hence

$$
g_{\Omega}(x, y) \geq \tilde{c}_{y} \rho(x) \geq-C_{8} \frac{\rho(x)}{\rho(y)}|\log (-\rho(y))| .
$$

The following is the key step in proving Theorem 2. The main idea comes from [3].

Proposition 5. There is a constant $C>0$ such that for any $y \in \Omega$ with $|\rho(y)|<$ $e^{-1}$ one has

$$
\begin{aligned}
& \left\{x \in \Omega: g_{\Omega}(x, y)<-1\right\} \\
\subset \quad & \left\{x \in \Omega: C^{-1}|\rho(y)| \cdot|\log (-\rho(y))|^{-1} \leq|\rho(x)| \leq C|\rho(y)| \cdot|\log (-\rho(y))|^{n}\right\} .
\end{aligned}
$$

Proof. From [2], we know that for any nonnegative psh functions $u, v$ defined on a smooth bounded domain $D$ in a Stein manifold with $\left.u\right|_{\partial D}=0$, then

$$
\int_{D}|u|^{n}\left(d d^{c} v\right)^{n} \leq n !\|v\|_{\infty}^{n-1} \int_{D}|v|\left(d d^{c} u\right)^{n}
$$

where $d^{c}=i(\bar{\partial}-\partial)$. Fix arbitrary $x, y \in \Omega$ with $\rho(x) \leq 2 \rho(y)$. Set $\epsilon=|\rho(y)|^{e}$ and $\alpha=-\frac{2}{\tau}(b+1) \log \epsilon$. We exhaust $\Omega$ by a sequence of smooth strongly pseudoconvex domains $\Omega_{j}, j=1,2, \cdots$. By the above inequality, we have

$$
\begin{aligned}
& \int_{\Omega_{j}}\left|g_{\Omega_{j}}(\cdot, y)\right|^{n}\left(d d^{c} \max \left\{g_{\Omega_{j}}(\cdot, x),-\alpha\right\}\right)^{n} \\
\leq & n ! \alpha^{n-1} \int_{\Omega_{j}}\left|\max \left\{g_{\Omega_{j}}(\cdot, x),-\alpha\right\}\right|\left(d d^{c} g_{\Omega_{j}}(\cdot, y)\right)^{n} \\
\leq & n !(2 \pi)^{n} \alpha^{n-1}\left|g_{\Omega_{j}}(y, x)\right|
\end{aligned}
$$

since $\left(d d^{c} g_{\Omega_{j}}(\cdot, y)\right)^{n}=(2 \pi)^{n} \delta_{y}$ (cf. [8]). It is also known from [8] that the measure $\left(d d^{c} \max \left\{g_{\Omega_{j}}(\cdot, x),-\alpha\right\}\right)^{n}$ is supported on $\left\{g_{\Omega_{j}}(\cdot, x)=-\alpha\right\}$ with total mass $(2 \pi)^{n}$. 
Hence

$$
\begin{aligned}
\inf _{\left\{g_{\Omega}(\cdot, x)=-\alpha\right\}}\left|g_{\Omega}(\cdot, y)\right|^{n} & \leftarrow \inf _{\left\{g_{\Omega_{j}}(\cdot, x)=-\alpha\right\}}\left|g_{\Omega_{j}}(\cdot, y)\right|^{n} \\
& \leq n ! \alpha^{n-1}\left|g_{\Omega_{j}}(y, x)\right| \\
& \rightarrow n ! \alpha^{n-1}\left|g_{\Omega}(y, x)\right|
\end{aligned}
$$

as $j \rightarrow \infty$. According to Lemma 4, one has $g_{\Omega}(z, x)>-1$ provided $|\rho(z)| \leq|\rho(x)|^{e}$. On the other hand, for any $z, x \in \Omega$ with $|\rho(z)|>|\rho(x)|^{e}$ one has

$$
\begin{aligned}
g_{\Omega}(z, x) & \geq \mu_{x}-C_{7}=\lambda_{x}-C_{7} \\
& \geq \varphi_{x}(z)-\log \left(-\phi_{x}(z)\right)+2 b e \log |\rho(x)|-C_{7} \\
& \geq \varphi_{x}(z)-\log \left(-\phi_{x}(z)\right)+\frac{2 b}{\tau} \log \epsilon-C_{8},
\end{aligned}
$$

which implies

$$
\left\{g_{\Omega}(\cdot, x)=-\alpha\right\} \subset B(x, \epsilon):=\left\{d_{F S}(\cdot, x)<\epsilon^{1 / \tau}\right\}
$$

provided $\epsilon$ is sufficiently small. Hence, by (1) there exists $\widetilde{x} \in B\left(x, \epsilon^{1 / \tau}\right)$ such that

$$
\left|g_{\Omega}(\widetilde{x}, y)\right|^{n} \leq C_{9}|\log \epsilon|^{n-1}\left|g_{\Omega}(y, x)\right| .
$$

By Bertini's Lemma, one can take a generic hyperplane $H$ such that it does not contain $x, \tilde{x}, y$. On $\mathbf{P}^{n} \backslash H$ one can introduce inhomogeneous coordinates $w=$ $\left(w_{1}, \cdots, w_{n}\right)$. We can also choose $H$ such that $|w(x)-w(\tilde{x})| \approx d_{F S}(x, \tilde{x})$ where the implicit constants depend only on $\Omega$. One can regard $\Omega \backslash H$ as an unbounded $C^{2}$ pseudoconvex domain in $\mathbf{C}^{n}$. Set

$$
\widetilde{\Omega}=\{w \in \Omega \backslash H: w+w(\widetilde{x})-w(x) \in \Omega \backslash H\} .
$$

Since $d s_{F S}^{2}=\partial \bar{\partial} \log \left(1+|w|^{2}\right) \leq \partial \bar{\partial}|w|^{2}$ on $\mathbf{P}^{n} \backslash H$, there is a constant $C_{10}>0$ such that

$$
\partial \widetilde{\Omega} \cap(\Omega \backslash H) \subset\left\{\delta_{\Omega}<C_{10} \epsilon^{1 / \tau}\right\} .
$$

Therefore,

$$
h(w)= \begin{cases}\max \left\{g_{\Omega}(w, w(y)), g_{\Omega}(w+w(\widetilde{x})-w(x), w(y))-\delta\right\}, & w \in \widetilde{\Omega}, \\ g_{\Omega}(w, w(y)), & w \in(\Omega \backslash H) \backslash \widetilde{\Omega},\end{cases}
$$

where $\delta=\sup _{\delta_{\Omega}<C_{10} \epsilon^{1 / \tau}}\left|g_{\Omega}(\cdot, w(y))\right|$ is a well-defined negative psh function with a logarithmic pole at $w(y)$ on $\Omega \backslash H$. Since $H$ is an analytic subset, $h$ extends to a psh function on the whole of $\Omega$. Therefore,

$$
g_{\Omega}(x, y) \geq h(w(x)) \geq g_{\Omega}(\widetilde{x}, y)-\delta .
$$

By (2), (3), for any $x, y \in \Omega$ with $\rho(x) \leq 2 \rho(y)$, one has

$$
\begin{aligned}
\left|g_{\Omega}(x, y)\right| & \leq \delta+C_{11}|\log \epsilon|^{1-\frac{1}{n}}\left|g_{\Omega}(y, x)\right|^{1 / n} \\
& \leq \frac{1}{2}+C_{12}\left(\frac{\rho(y)}{\rho(x)}\right)^{1 / n}|\log (-\rho(y))|
\end{aligned}
$$

according to Lemma 4 . The proof is complete.

Proof of Theorem 2. We follow the argument as in [10]. Let $y_{1}, y_{2} \in \Omega$ be two arbitrary points satisfying

$$
\left|\rho\left(y_{2}\right)\right|<2^{-e}, C\left|\rho\left(y_{1}\right)\right| \cdot\left|\log \left(-\rho\left(y_{1}\right)\right)\right|^{n} \leq C^{-1}\left|\rho\left(y_{2}\right)\right| \cdot\left|\log \left(-\rho\left(y_{2}\right)\right)\right|^{-1} .
$$


We take a complete orthonormal basis $\left\{h_{j}\right\}_{j=0}^{\infty}$ for $\mathcal{H}$ such that $h_{j}\left(y_{2}\right)=0$ for all $j \geq 1$. According to Kobayashi [15, we can immerse $M$ into the infinite-dimensional complex projective space $\mathbf{C P}(\mathcal{H})$ via the map

$$
\sigma: x \mapsto\left(h_{0}(x): h_{1}(x): \cdots\right) .
$$

Since each point $P=\left(\zeta_{0}: \zeta_{1}: \cdots\right)$ in the projective space corresponds to an entire great circle of the unit sphere consisting of points $\left(\zeta_{0} e^{i \theta}, \zeta_{1} e^{i \theta}, \cdots\right)$, then the FubiniStudy distance between two points $P, Q$ is equal to the distance in the spherical geometry between the corresponding great circles. By the choice of the basis, we have $\sigma\left(y_{2}\right)=(1: 0: \cdots)$ and $\sigma\left(y_{1}\right)=\left(a_{0}: a_{1}: \cdots\right)$ where $a_{j}=h_{j}^{*}\left(y_{1}\right) / \sqrt{K_{\Omega}^{*}\left(y_{1}\right)}$. Hence,

$$
\begin{aligned}
\operatorname{dist}_{\Omega}\left(y_{1}, y_{2}\right) & \geq \operatorname{dist}_{F S}\left(\sigma\left(y_{1}\right), \sigma\left(y_{2}\right)\right) \\
& \geq \inf _{\theta_{1}, \theta_{2}}\left|e^{i \theta_{1}}\left(a_{0}, a_{1}, \cdots\right)-e^{i \theta_{2}}(1,0, \cdots)\right| \\
& =\sqrt{\left(1-\left|a_{0}\right|\right)^{2}+\sum_{j=1}^{\infty}\left|a_{j}\right|^{2}} .
\end{aligned}
$$

Therefore, if $\left|a_{0}\right| \leq 1 / 2$, then $\operatorname{dist}_{\beta}\left(y_{1}, y_{2}\right) \geq 1 / 2$. Otherwise, take a smooth function $\lambda$ on $\mathbf{R}$ such that $\lambda=1$ on $(-\infty,-1]$ and $\lambda=0$ on $[0, \infty)$. Set

$$
\begin{aligned}
& \eta=\lambda\left(-\log \left(-g_{\Omega}\left(\cdot, y_{1}\right)+1\right)+\log 2\right) h_{0}, \\
& \varphi=2 n\left(g_{\Omega}\left(\cdot, y_{1}\right)+g_{\Omega}\left(\cdot, y_{2}\right)\right)-\log \left(-g_{\Omega}\left(\cdot, y_{1}\right)+1\right) .
\end{aligned}
$$

By Proposition 5, we see that $\left\{g_{\Omega}\left(\cdot, y_{1}\right)<-1\right\} \cap\left\{g_{\Omega}\left(\cdot, y_{2}\right)<-1\right\}=\emptyset$. By the well-known $L^{2}$ estimates (cf. [7], [16]), we can solve the equation $\bar{\partial} u=\bar{\partial} \eta$ in such a way that

$$
\begin{aligned}
\left|\int_{\Omega} u \wedge \bar{u} e^{-\varphi}\right| & \leq\left.\left|\int_{\Omega}\right| \bar{\partial} \lambda\right|_{\partial \bar{\partial} \varphi} ^{2} h_{0} \wedge \bar{h}_{0} e^{-\varphi} \mid \\
& \leq C_{12}
\end{aligned}
$$

since $\partial \bar{\partial} \varphi \geq\left(-g_{\Omega}\left(\cdot, y_{1}\right)+1\right)^{-2} \partial g_{\Omega}\left(\cdot, y_{1}\right) \bar{\partial} g_{\Omega}\left(\cdot, y_{1}\right)$ holds in the sense of distribution. Therefore, $F=\eta-u$ is holomorphic on $\Omega$ and satisfies $F\left(y_{1}\right)=h_{0}\left(y_{1}\right), F\left(y_{2}\right)=0$ and

$$
\left|\int_{\Omega} F \wedge \bar{F}\right| \leq C_{13}
$$

Hence

$$
\begin{gathered}
\operatorname{dist}_{\Omega}\left(y_{1}, y_{2}\right) \geq \sqrt{\sum_{j=1}^{\infty}\left|a_{j}\right|^{2}} \geq \sqrt{\frac{F\left(y_{1}\right) \wedge \bar{F}\left(y_{1}\right)}{C_{13} K_{\Omega}\left(y_{1}\right)}} \\
=\sqrt{\frac{h_{0}\left(y_{1}\right) \wedge \bar{h}_{0}\left(y_{1}\right)}{C_{13} K_{\Omega}\left(y_{1}\right)}}=\frac{\left|a_{0}\right|}{\sqrt{C_{13}}} \geq \frac{1}{2 \sqrt{C_{13}}} .
\end{gathered}
$$

Now if $c_{0}, c_{1}, \cdots, c_{k}$ are finite increasing positive numbers such that $c_{k} \leq 2^{-e}$ and

$$
C^{-1} c_{k}\left|\log c_{k}\right|^{-1}=C c_{k-1}\left|\log c_{k-1}\right|^{n},
$$

then

$$
c_{k} \leq C^{2} c_{k-1}\left|\log c_{k-1}\right|^{n} \leq C^{4} c_{k-2}\left|\log c_{k-2}\right|^{2 n} \leq \cdots \leq C^{2 k} c_{0}\left|\log c_{0}\right|^{n k} .
$$


Given $y \in \Omega$, fix a point $y_{0}$ with $\left|\rho\left(y_{0}\right)\right|=2^{-e}$. Take a Bergman geodesic $l$ connecting $y_{0}, y$. Let $c_{0}=|\rho(y)|, c_{k}=2^{-e}$. Take $y_{i} \in l$ with $\left|\rho\left(y_{i}\right)\right|=c_{i}, i=$ $0,1, \cdots, k$. Then

$$
\operatorname{dist}_{\Omega}\left(y_{0}, y\right) \geq \sum_{i=0}^{k-1} \operatorname{dist}_{\Omega}\left(y_{i}, y_{i+1}\right) \geq C_{14} k,
$$

from which the desired estimate follows.

\section{Proof of Theorem 1}

Let $y \in \Omega \cap V$ be an arbitrary point with $|\rho(y)|<e^{-1}$. Set $\tilde{\epsilon}=C^{-1}|\rho(y)|$. $|\log (-\rho(y))|^{-1}$ and $\Omega_{\tilde{\epsilon}}=\{x \in \Omega:|\rho(x)|>\tilde{\epsilon}\}$. Here $C$ is the constant in Proposition 5. Set

$$
\begin{aligned}
& \tilde{\lambda}_{y}=\varphi_{y}-\log \left(-\phi_{y}\right)-b \log (-\rho)+b \log \tilde{\epsilon}, \\
& \psi_{y}=\max \left\{\tilde{\lambda}_{y}, g_{\Omega}(\cdot, y)\right\} .
\end{aligned}
$$

Then $\psi_{y}$ is a negative psh function with a logarithmic pole at $y$ on $\Omega_{\tilde{\epsilon}}$ such that

$$
\begin{aligned}
& \left\{x \in \Omega_{\tilde{\epsilon}}: \psi_{y}(x)<-1\right\} \subset\left\{x \in \Omega_{\tilde{\epsilon}}: g_{\Omega}(x, y)<-1\right\} \\
& \subset\left\{x \in \Omega_{\tilde{\epsilon}}:|\rho(x)| \leq C|\rho(y)| \cdot|\log (-\rho(y))|^{n}\right\} .
\end{aligned}
$$

On the other hand, for any $0<\tilde{r}<r_{0}$, there is a positive constant $\tilde{C}$ depending only on $\tilde{r}$ such that

$$
\psi_{y}(x) \geq \tilde{\lambda}_{y}(x) \geq-\tilde{C}-b(n+1) \log |\log (-\rho(y))|, \quad \forall x \in\left\{\psi_{y}<-1\right\} \backslash B(y, \tilde{r}) .
$$

Without loss of generality, we assume that $B(y, 2 \tilde{r}) \subset U$. Choose a holomorphic $n$-form $f$ on $\Omega \cap U$ with unit $L^{2}$-norm such that $f \wedge \bar{f}(y)=K_{\Omega \cap U}(y)$. Let $\kappa, \lambda$ be the cut-off functions as above. Set

$$
\begin{aligned}
\tilde{\varphi}_{y} & =2 n \psi_{y}-\log \left(-\psi_{y}+1\right), \\
v & =\bar{\partial}\left(\lambda\left(-\log \left(-\psi_{y}+1\right)+\log 2\right) \kappa\left(d_{F S}(\cdot, y) / 2 \tilde{r}\right) f\right)
\end{aligned}
$$

on $\Omega_{\tilde{\epsilon}}$. We recall the following $L^{2}$ estimate:

Theorem (cf. [10, 1]). Let $M$ be a Stein manifold. Let $\varphi, \psi$ be psh functions such that $r \partial \bar{\partial} \psi \geq \partial \psi \bar{\partial} \psi$ holds in the sense of distribution for some $0<r<1$. Then for any $\bar{\partial}$-closed $(n, 1)$ form $v$ with $\int_{M}|v|_{\partial \bar{\partial}(\varphi+\psi)}^{2} e^{\psi-\varphi}<+\infty$, there exists an $(n, 0)$ form $u$ on $M$ such that $\bar{\partial} u=v$ and

$$
\left|\int_{M} u \wedge \bar{u} e^{\psi-\varphi}\right| \leq C_{r} \int_{M}|v|_{\partial \bar{\partial}(\varphi+\psi)}^{2} e^{\psi-\varphi} .
$$

We apply this theorem with $\psi=-\frac{1}{2} \log (-\rho), \varphi=\tilde{\varphi}_{y}+\psi$ to get a solution $u$ of $\bar{\partial} u=v$ such that

$$
\left|\int_{\Omega_{\tilde{\epsilon}}} u \wedge \bar{u} e^{-\tilde{\varphi}_{y}}\right| \leq \tilde{C}_{2}|\log (-\rho(y))|^{\gamma} \leq \tilde{C}_{3}\left|\log \delta_{\Omega}(y)\right|^{\gamma}
$$

because of (4), (5) and

$$
\partial \bar{\partial}\left(\tilde{\varphi}_{y}+\psi\right) \geq \partial \log \left(-\psi_{y}+1\right) \bar{\partial} \log \left(-\psi_{y}+1\right)+\frac{C_{1}}{2} d s_{F S}^{2}
$$


Here $\gamma>0$ depends only on $b, n$ and $\tilde{C}_{2}, \tilde{C}_{3}$ are independent of $y$. Thus we obtain a holomorphic $n$-form

$$
F=\lambda\left(-\log \left(-\psi_{y}+1\right)+\log 2\right) \kappa\left(d_{F S}(\cdot, y) / 2 \tilde{r}\right) f-u
$$

on $\Omega_{\tilde{\epsilon}}$ such that $F \wedge \bar{F}(y)=K_{\Omega \cap U}(y)$ and its $L^{2}$-norm is bounded above by a constant multiple of $\left|\log \delta_{\Omega}(y)\right|^{\gamma}$. Finally, we apply the above theorem with

$$
\begin{aligned}
\psi & =-\frac{1}{2} \log \left(-g_{\Omega}(\cdot, y)+1\right) \\
\varphi & =2 n g_{\Omega}(\cdot, y)+\psi
\end{aligned}
$$

to get a solution of

$$
\bar{\partial} u=v:=\bar{\partial}\left(\lambda\left(-\log \left(-g_{\Omega}(\cdot, y)+1\right)+\log 2\right) F\right)
$$

on $\Omega$ such that

$$
\left|\int_{\Omega} u \wedge \bar{u} e^{-2 n g_{\Omega}(\cdot, y)}\right| \leq \tilde{C}_{4}\left|\int_{\Omega_{\tilde{\epsilon}}} F \wedge \bar{F}\right|
$$

Set $\tilde{F}=\lambda\left(-\log \left(-g_{\Omega}(\cdot, y)+1\right)+\log 2\right) F-u$. Then $\tilde{F}$ is a holomorphic $n$-form on $\Omega$ satisfying $\tilde{F}(y)=F(y)$ and the $L^{2}$-norm bounded above by a constant multiple of $\left|\log \delta_{\Omega}(y)\right|^{\gamma}$, from which we obtain the estimate of the Bergman kernel. The argument for the Bergman metric is similar.

\section{ACKNOWLEDGEMEnTS}

The author thanks Professor Takeo Ohsawa for posing the question and to the Graduate School of Mathematics, Nagoya University for its hospitality. He also thanks the referee for his/her careful reading and valuable remarks on the manuscript.

\section{REFERENCES}

[1] B. Berndtsson and Ph. Charpentier, A Sobolev mapping property of the Bergman kernel, Math. Z. 235 (2000), 1-10. MR.1785069 (2002a:32039)

[2] Z. Blocki, Estimates for the complex Monge-Ampère operator, Bull. Pol. Acad. Sci. 41 (1993), 151-157. MR1414762 (97j:32009)

[3] - - The Bergman metric and the pluricomplex Green function, MPI (Leipzig) preprint no: 85 (2002).

[4] Z. Blocki and P. Pflug, Hyperconvexity and Bergman completeness, Nagoya Math. J. 151 (1998), 221-225. MR1650305 (2000b:32065)

[5] B. Y. Chen, Bergman completeness of hyperconvex manifolds, preprint.

[6] B. Y. Chen and J. H. Zhang, The Bergman metric on a Stein manifold with a bounded plurisubharmonic function, Trans. Amer. Math. Soc. 354 (2002), 2997-3009. MR1897387 (2003c:32014)

[7] J. P. Demailly, Estimations $L^{2}$ pour l'opérateur d'un fibré vectoriel holomorphe semi-positiv au dessus d'une variété kählérienne complète, Ann. Sci. Éc. Norm. Sup. 15 (1982), 457-511. MR0690650 (85d:32057)

[8] —-, Mesures de Monge-Ampère et mesures pluriharmoniques, Math. Z. 194 (1987), 519-564. MR0881709 (88g:32034)

[9] K. Diederich, J. E. Fornaess and G. Herbort, Boundary behavior of the Bergman metric, Complex Analysis of Several Variables (Madison, Wis., 1982), 59-67, Proc. Sympos. Pure Math. 41, Amer. Math. Soc., Providence, RI, 1984. MR0740872 (85j:32039)

[10] K. Diederich and T. Ohsawa, An estimate for the Bergman distance on pseudoconvex domains, Ann. of Math. 141 (1995), 181-190. MR1314035(95j:32039)

[11] —-, On pseudoconvex domains in $\mathbf{P}^{n}$, Tokyo J. Math. 21 (1998), 353-358. MR1663574 (99k:32024) 
[12] R. E. Greene and H. Wu, Function theory on manifolds which possess a pole, Lecture Notes in Mathematics 699, Springer-Verlag, 1979. MR0521983 (81a:53002)

[13] G. Herbort, The Bergman metric on hyperconvex domains, Math. Z. 232 (1999), 183-196. MR.1714284 (2000i:32020)

[14] M. Jarnicki and P. Pflug, Bergman completeness of complete circular domains, Ann. Pol. Math. Vol 50 (1989), 219-222. MR1044868 (91f:32026)

[15] S. Kobayashi, Geometry of bounded domains, Trans. Amer. Math. Soc. 92 (1959), 267-290. MR0112162(22:3017)

[16] T. Ohsawa, Boundary behavior of the Bergman kernel function on pseudoconvex domains, Publ. RIMS, Kyoto Univ. 20 (1984), 897-902. MR0764336 (86d:32025)

[17] - - On the completeness of the Bergman metric, Proc. Jap. Acad. Sci. 57, Ser. A (1981), 238-240. MR0618233 (82j:32053)

[18] T. Ohsawa and N. Sibony, Bounded p.s.h. functions and pseudoconvexity in Kähler manifolds, Nagoya Math. J. 149 (1998), 1-8. MR1619572 (2000b:32062)

[19] T. Ohsawa and K. Takegoshi, On the extension of $L^{2}$ holomorphic functions, Math. Z. 195 (1987), 197-204. MR0892051 (88g:32029)

[20] W. Zwonek, Completeness, Reinhardt domains and the method of complex geodesics in the theory of invariant functions, Dissert. Math. 388 (2000), 103 pp. MR1785672(2001h:32016)

Department of Applied Mathematics, Tongui University, Shanghai 200092, Peoples Republic of China

Current address: Graduate School of Mathematics, Nagoya University, Chikusa-ku, Nagoya 464-8602, Japan

E-mail address: by-chen@math.nagoya-u.ac.jp 